\title{
Uma proposta pedagógica para o ensino de potenciação por meio de atividades lúdicas
}

\author{
Daniely Cristiny Lucas Reghim Glaucia Maria Bressan
}

\begin{abstract}
Resumo
O presente trabalho apresenta uma proposta pedagógica de ensino que contribua para a abordagem do conteúdo de Potenciação no $1^{\circ}$ ano do Ensino Médio, mediante a inserção de jogos e atividades lúdicas no cotidiano escolar. A proposta foi aplicada em uma turma de uma escola pública do interior do estado do Paraná, onde foi realizado o Estágio Supervisionado da primeira autora deste trabalho. Foi aplicado um jogo de bingo referente ao tema trabalhado, abordando o conteúdo de Potenciação. A metodologia aplicada trouxe resultados positivos na realização da tarefa dos alunos, proporcionando dinamismo, desafios e uma maior interação entre os alunos, contribuindo assim para uma melhor aprendizagem.
\end{abstract}

Palavras-chave: Potenciação; Jogos; Regência; Ensino Médio.

\section{Abstract}

This paper presents a pedagogical proposal of teaching, which contributes to the approach of the Potentiation content in the first year of high school, through the insertion of games and recreational activities in daily school life. The proposal was applied in a class of a public school in the interior of the state of Paraná, where the Supervised Internship of the first author of this paper was performed. A bingo game was applied, corresponding to the taught subject, addressing the content of Potentiation. The applied methodology brought positive results in the accomplishment of the students task, providing dynamism, challenges and greater interaction among the students, thus contributing to a better learning.

Keywords: Potentiation; Games; Regency; High school.

\section{Introdução}

O conceito de Potenciação está presente hoje em diversas situações do cotidiano da sociedade, sendo utilizado, por exemplo, nos cálculos envolvendo juros compostos, que são desenvolvidos baseado na potenciação das taxas de juros; a função exponencial também é um exemplo onde utilizamos potências, a notação científica utiliza potências no intuito de representar números muito grandes ou pequenos. É notória a importância das potências nos cálculos matemáticos modernos, facilitando e contribuindo na resolução de problemas cotidianos. 
Em se tratando de aulas de matemática, o uso de jogos implica uma transformação significativa nos processos de ensino e aprendizagem que permite modificar-se o modelo tradicional de ensino, que muitas vezes tem no livro e em exercícios padronizados seu principal recurso didático [8]. O trabalho com jogos nas aulas de matemática, quando bem planejado e orientado, auxilia o desenvolvimento de habilidades como observação, análise, levantamento de hipóteses, busca de suposições, reflexão, tomada de decisões, argumentação e organização, as quais estão estreitamente relacionadas ao assim chamado raciocínio lógico [?]. Além disso, para o ensino de matemática por meio de jogos, é fundamental o domínio dos conceitos pelo docente, para que as dúvidas que possam surgir ao decorrer do jogo sejam atendidas. Conforme [6], as formas de manipular valores, realizar operações, elaborar e solucionar problemas são determinados não só pela estrutura lúdica, mas também pelos conhecimentos socioculturais que, incorporados pelas crianças, passam a fazer parte do sistema de regras dos jogos.

Neste trabalho, propõe-se a utilização de jogos e atividades lúdicas para a abordagem da definição e das propriedades da potenciação para relembrar o conteúdo já visto no ensino fundamental, pois sua relação direta com operações elementares da matemática permite simplificar expressões mais complexas para melhor manipulá-las e, mesmo no Ensino Médio, tornam-se conteúdos importantes para as disciplinas de física, química, biologia e para a própria matemática. "A aplicação do jogo, trazendo situações do contexto do/a aluno/a, vem contemplar toda a sua gama de conhecimento que foi construída fora da escola e, muitas vezes, é ignorada em sala de aula' '[5]. Com o intuito de aproveitar esta bagagem de conhecimento trazida e tornar o aprendizado mais lúdico, buscou-se a utilização de jogos matemáticos para contribuir na aprendizagem da Potenciação no $1^{\mathrm{O}}$ ano do ensino médio, proporcionando dinamismo, desafios, uma maior interação entre os alunos e entre esses e o professor, além de desenvolver vários aspectos atrelados à aprendizagem. A atividade proposta foi aplicada em uma turma de uma escola pública do interior do estado do Paraná, onde foi realizado o Estágio Supervisionado da primeira autora deste trabalho.

A proposta apresentada neste trabalho mostra-se pertinente pois, de acordo com os Parâmetros Curriculares Nacionais [1], "embora o estudo dos números e das operações seja um tema importante nos currículos do ensino fundamental, constata-se, com frequência, que muitos alunos chegam ao final desse curso com um conhecimento insuficiente dos números, de como eles são utilizados e sem ter desenvolvido uma ampla compreensão dos diferentes significados das operações". Desta forma, o uso de jogos se torna uma ferramenta de auxílio para progressão do aprendizado do aluno.

\section{Atividade Desenvolvida no $1^{\circ}$ ano do Ensino Médio}

Primeiramente, as atividades do Estágio Supervisionado foram iniciadas a partir das observações em sala de aula, no $1^{\circ}$ do Ensino Médio. Em seguida, a regência ocorreu nos meses de setembro, outubro e novembro de 2018. Foi realizada uma revisão sobre o conteúdo de potenciação com a aplicação de uma lista de resolução de problemas. Logo depois, foi exposto o conteúdo com as propriedades e as regras da potenciação.

Em seguida, foi aplicado um jogo de Bingo com as propriedades da Potenciação, por já terem visto esse conteúdo em outro ano escolar e por ter feito mais uma revisão dada na aula anterior; assim, foram aplicadas algumas expressões mais elaboradas. A Figura 1 exibe a relação das expressões utilizadas para o jogo de Bingo. Essas expressões foram escritas no quadro, uma a uma, baseadas em $[2,3]$. Os alunos deveriam então calcular a expressão em seu caderno e obter o resultado da operação. Em seguida, deveriam verificar se aquele resultado constava em sua cartela, recebida pela 
professora. Em caso afirmativo, marcavam um sinal sobre o valor; em caso negativo, aguardavam a próxima expressão. O aluno que completasse a cartela toda, seria o vencedor [4]. Uma das cartelas distribuídas para os alunos é exibida na Figura 2.

\begin{tabular}{|c|c|c|c|c|c|}
\hline$\frac{10^{-2} \cdot 10^{-3} \cdot 10^{-4}}{10^{-1} \cdot 10^{-6}}$ & $\frac{2 .\left(3^{6}+3^{5}\right)}{3^{4}-3^{3}}$ & $7^{-2}$ & $\begin{array}{c}{\left[\left(-\frac{1}{2}\right)^{4}:\left(-\frac{1}{2}\right)^{3}\right] \cdot\left(-\frac{1}{2}\right)^{6}} \\
+2^{-7}\end{array}$ & $\frac{8^{-2}-4^{-2}}{8^{-1}-4^{-1}}$ & {$[-2]^{2}-2^{-1}$} \\
\hline$(0,3)^{-1}+(-27)^{0,333 \ldots}$ & 2. $10^{-1}+3.2^{-2}$ & $4^{0}+4^{-1}-5^{-1}$ & 4. $(2,5)^{2}-8 \cdot(1,5)^{2}$ & $(0,4)^{2}+2 \cdot(1,2)^{2}$ & $y=\frac{2^{-2}+2^{2}-2^{-1}}{2^{-2}-2^{-1}}$ \\
\hline$(-1)^{10}+3 \cdot(-1)^{5}-3 \cdot(-1)^{6}$ & $8^{0,666 \ldots}-9^{0,5}$ & {$\left[16^{2} .64^{3}\right]: 1024$} & $\frac{6 \cdot 10^{-3} \cdot 10^{-4} \cdot 10^{8}}{6 \cdot 10^{-1} \cdot 10^{4}}$ & $\begin{array}{c}10 x^{2}+100 x-100 \\
\quad \operatorname{para} x=5\end{array}$ & $\begin{array}{l}\text { Se } a=3^{2} \text { e } b=a^{2} \\
\text { então o valor do } \\
\text { produto ab é }\end{array}$ \\
\hline $\begin{array}{l}x^{3}-x^{2}-x+1 \\
\text { para } x=-1\end{array}$ & $\frac{2^{3+x}-3^{x-3}}{2^{x}+2^{x-3}}$ & $\frac{3^{x+2}+3^{x+1}}{3^{x-1}}$ & $\frac{35^{-1} \cdot 40^{-1} \cdot 10^{-2} \cdot 5 \cdot 100}{2^{3} \cdot 14^{-1} \cdot 5 \cdot 25}$ & $\begin{aligned} & {\left[4^{2}+(5-3)^{2}\right]: }(9 \\
&-7)^{2}\end{aligned}$ & $\left(5^{-5}\right)^{5}$ \\
\hline $\begin{array}{l}\frac{\left(\frac{x^{3}-25 x}{3 x-15}\right) \cdot\left(\frac{3 y}{x}\right)}{\frac{x y+5 y}{4}} \\
\text { Para } x=2,71 \text { e } y=3,14\end{array}$ & $\begin{array}{c}\frac{x^{2} y^{2}-x^{3} y}{y^{2}-x^{2}} \\
\text { Para } x=0,5 \text { e } y=1,5\end{array}$ & $\frac{4^{2}-4^{-2}}{4^{2}+4^{-2}}$ & $5^{1} \cdot 3^{-2}+3^{-1}-3 \cdot 3^{0}$ & {$\left[2^{9}:\left(2^{2} \cdot 2\right)^{3}\right]^{-3}$} & $\begin{array}{r}(-5)^{2}-3^{2}+\left(\frac{2}{3}\right)^{0}:\left(3^{-2}\right. \\
\left.+\frac{1}{5}+\frac{1}{2}\right)\end{array}$ \\
\hline$(-1)^{3}+\left(-\frac{1}{2}\right)^{-1} \cdot(-2)^{-1}$ & $2^{3}-2.3^{2}$ & $\begin{array}{l}0^{25}+(-25)^{0} \\
+(-1)^{4}\end{array}$ & $(0,16)^{0}$ & $2^{2} \cdot 2^{-3}$ & $\frac{2^{2003} \cdot 9^{1001}}{4^{1001} \cdot 3^{2003}}+\frac{2^{2002} \cdot 9^{1001}}{4^{1001} \cdot 3^{2003}}$ \\
\hline$\frac{3^{0}+(-2)^{2}-\left(\frac{1}{3}\right)^{-1}}{\left(\frac{1}{2}\right)^{-2}}$ & $\frac{(-5)^{2}-4^{2}}{2^{-1}+2^{-2}}$ & $(5)^{4}-(-5)^{4}$ & $(-2)+(-3) \cdot(-2)^{-1}:(-3)$ & $9^{\frac{1}{2}}$ & $14^{1}$ \\
\hline$x=\left(-\frac{1}{3}\right)^{3}+\left[3^{-1}(-3)^{-1}\right]^{-2}$ & $3^{2}-23+\left(\frac{1}{2}\right)^{0}$ & $\left(\frac{3^{4} \cdot 2^{3}}{3^{7} \cdot 2^{2}}\right)^{-1}$ & $\left(27^{\frac{1}{3}}+64^{\frac{1}{2}}-8^{\frac{2}{3}}+4^{\frac{1}{2}}\right)^{\frac{1}{2}}$ & $\frac{2^{-1}+(-2)^{-1}-(-2)^{2}}{1-2^{-2}}$ & $\frac{9^{3} \cdot 27^{4} \cdot 3^{-7}}{3^{-1} \cdot 243^{2}}$ \\
\hline$\left(2^{2} \cdot 2^{-3} \cdot 3^{-1} \cdot 3^{2}\right)^{2}$ & $5.10^{8} \cdot 4 \cdot 10^{-3}$ & $\frac{10^{-1}}{5^{-1}+2^{-1}}$ & $(0,5)^{3}-(0,75)^{2}$ & $4 \cdot 2^{3}-\left(\frac{3}{2}\right) \cdot(-2)^{1}$ & $(0,7777 \ldots)^{0}$ \\
\hline$\frac{1}{(-2)^{-5}}$ & $-5^{3}$ & $\left(-\frac{2}{3}\right)^{-3}$ & $(-1)^{0}+(-6):(-2)-2^{4}$ & $\frac{\left(10^{-3} \cdot 10^{5}\right)}{\left(10.10^{4}\right)}$ & $\begin{array}{c}20 x^{3}+2 x^{2} y^{5} \\
\text { para, } x=-4 \text { e } y=2\end{array}$ \\
\hline
\end{tabular}

Figura 1: Expressões utilizadas no jogo de bingo.

Ao entrar em sala, os alunos foram receptivos e participativos durante quase todas as atividades, apresentando bons resultados nos trabalhos em grupos e individuais. O conteúdo aplicado, Potenciação, foi abordado de uma maneira que os alunos compreendessem, trazendo exercícios para fixação. As inúmeras dúvidas que surgiam foram vistas e sanadas com a aplicação da lista de exercícios, resoluções de problemas e jogos, mostrando-lhes que existem outras formas de aprendizagem além da que eles estão acostumados. Mas acreditamos que o ensino deve ser constante e que em apenas poucas aulas não conseguiríamos fazer com que esse conteúdo fosse totalmente compreendido. 


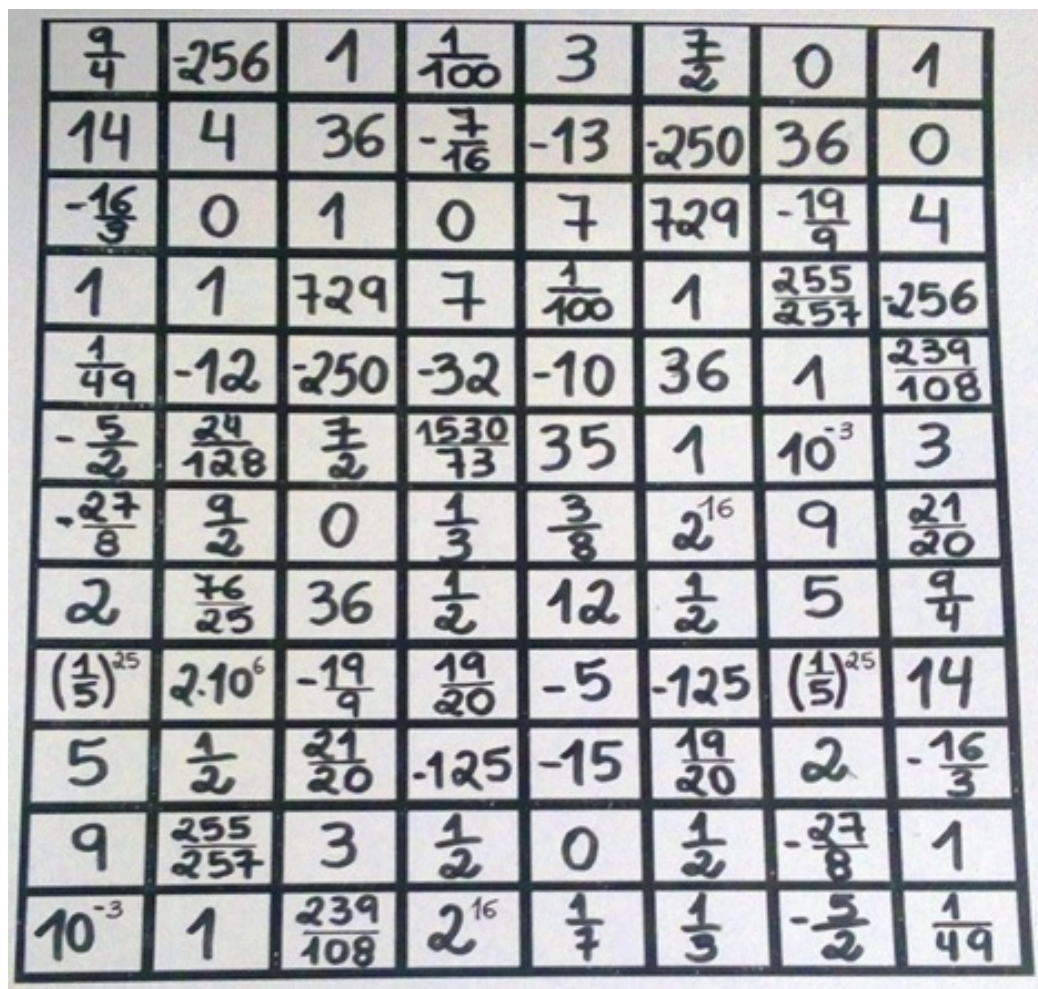

Figura 2: Exemplo de cartela distribuída aos alunos.

Em síntese, a metodologia aplicada trouxe resultados positivos na realização da tarefa dos alunos. Isso só foi possível com o apoio da professora supervisora, que nos deu total liberdade para desenvolver as atividades propostas e sugestões sobre como poderíamos proceder.

\section{Conclusão}

O conteúdo matemático foi trabalhado de forma lúdica, abrangendo a resolução de problemas e discussão sobre a adaptação da prática cotidiana em que todos tiveram conhecimentos obtidos; entretanto, fez-se necessário também o estudo sobre o conteúdo matemático abordado nos problemas.

Com essa experiência, percebemos que um dos fatores que deixa os professores insatisfeitos é a falta de disciplina e de interesse dos alunos. Precisamos, portanto, sempre buscar alternativas e novas metodologias para despertar o interesse, a atenção e também conquistar o respeito dos alunos, além de ter uma postura efetiva de um profissional que se preocupa verdadeiramente com o aprendizado, que deve exercer o papel de um mediador entre a sociedade e a particularidade do educando. Devemos despertar no educando a consciência de que ele não está pronto, aguçando-lhe o desejo de se complementar. Esse é o grande desafio que o educador encontra em sua profissão.

Acreditamos que experiências como essas são importantes no estágio, como parte da formação do 
professor, e também como uma forma de ensinar relacionando o conteúdo com situações problemas do dia a dia, mostrando ao aluno o quanto a construção do conhecimento é essencial para sua vida.

\section{Agradecimentos}

As autoras agradecem o Departamento Acadêmico de Matemática da UTFPR Campus Cornélio Procópio.

\section{Referências}

[1] Brasil. Ministério da Educação. Secretaria de Educação Fundamental. Parâmetros Curriculares Nacionais: Matemática. Brasília: MEC/SEF, 1998. 148 pp.

[2] Carvalho, A. Potenciação e Radiação lista 5. Disponível em: https://pt.slideshare.net/delimacarvalho/potenciaao-e-radiciaao-lista-5. Acesso em: 20 setembro de 2018.

[3] Ferreira, J. Reforço Orientado, Potenciação. Disponível em: https://saturniz.files.wordpress.com/2015/11/potenciac3a7c3a3o-9c2b0-ano.pdf. Acesso em: 20 de setembro 2018.

[4] Giordani, J. Bingo. Disponível em: https://euridespidibmatematica.wordpress.com/2015/04/28/bingo/: Acesso em: 12 de outubro 2019.

[5] Lara, I.C.M. Jogando com a Matemática de $5^{\underline{a}}$ a $8^{\underline{a}}$ série. São Paulo: Rêspel, 2003.

[6] Muniz, C.A. Brincar e jogar: enlaces teóricos e metodológicos no campo da educação matemática. São Paulo: Autêntica, 2010.

[7] Silva, M.N.P. Potenciação: a utilização de potências no cotidiano. Disponível em: https://mundoeducacao.bol.uol.com.br/matematica/a-utilizacao-potencias-no-cotidiano.htm. Acesso em: 12 de outubro 2019.

[8] Smole, K.S.; Diniz, M.I.; Milani, E. Cadernos de Mathema Jogos de matemática de $6^{o}$ a $9^{\circ}$ ano. Porto Alegre: artmed editora, 2007.

Daniely Cristiny Lucas Reghim

Universidade Tecnológica Federal do Paraná - câmpus Cornélio Procópio $<$ danielyreghim@alunos.utfpr.edu.br>

Glaucia Maria Bressan

Universidade Tecnológica Federal do Paraná - câmpus Cornélio Procópio <glauciabressan@utfpr.edu.br>

Recebido: $13 / 10 / 2019$

Publicado: 13/12/2019 